18

\title{
Advanced Nanotools for Imaging of Solid Tumors and Circulating and Disseminated Cancer Cells*
}

\author{
(C) A. Sukhanova ${ }^{1,2}$, F. Ramos-Gomes ${ }^{3}$, F. Alves ${ }^{3,4}$, P. Chames ${ }^{5}$, D. Baty ${ }^{5}$, and I. Nabiev ${ }^{1,29}$ \\ ${ }^{1}$ Laboratory of Nano-Bioengineering, National Research Nuclear University MEPhl (Moscow Engineering Physics Institute), \\ 115409 Moscow, Russia \\ ${ }^{2}$ Laboratoire de Recherche en Nanosciences, LRN-EA4682, Ulniversité de Reims Champagne-Ardenne, \\ 51100 Reims, France \\ ${ }^{3}$ Translational Molecular Imaging Unit, Max Planck Institute for Experimental Medicine, \\ 37075 Göttingen, Germany \\ ${ }^{4}$ Clinic of Haematology and Medical Oncology, University Medical Center Göttingen, \\ 37075 Göttingen, Germany \\ ${ }^{5}$ Aix Marseille University, CNRS, INSERM, Institut Paoli-Calmettes, Centre de Recherche Contre le Cancer de Marseille (CRCM), \\ 13009 Marseille, France \\ ؟e-mail: igor.nabiev@univ-reims.fr
}

Received July 6, 2018

Semiconductor quantum dots (QDs) are characterized by orders of magnitude higher multiphoton linear absorption cross-sections compared with conventional organic dyes. Combined with the QD photoluminescence quantum yield approaching $100 \%$ and their rock-solid photostability, this fact opens great prospects for the twophoton functional tumor imaging with QDs tagged with highly specific recognition molecules. Single-domain antibodies (sdAbs) or „nanobodies“ derived from lamas are the smallest high-affinity recognition molecules, which may be tagged with the QDs thus permitting not only solid tumors multiphoton imaging but also rare disseminated cancer cells and micrometastases in the depth of the tissue to be detected.

DOI: $10.21883 /$ OS.2018.11.46828.209-18

\footnotetext{
* International Conference „PCNSPA 2018 - Photonic Colloidal Nanostructures: Synthesis, Properties, and Applications", Saint Petersburg, Russia, June 4-8, 2018.

Полный текст статьи опубликован в английской версии журнала.
} 